\title{
Avaliação dos usuários sobre as farmácias públicas no Brasil
}

\author{
User evaluation of public pharmacy services in Brazil
}

\author{
Karen Sarmento Costa (http://orcid.org/0000-0002-2218-6024) ${ }^{1}$ \\ Anamaria Vargas Zaccolo (https://orcid.org/0000-0001-6195-9301) ${ }^{1}$ \\ Noemia Urruth Leão Tavares (https://orcid.org/0000-0001-6180-7527) ${ }^{2}$ \\ Paulo Sérgio Dourado Arrais (https://orcid.org/0000-0002-4502-8467) ${ }^{3}$ \\ Vera Lucia Luiza (https://orcid.org/0000-0001-6245-7522) ${ }^{4}$ \\ Maria Auxiliadora Oliveira (https://orcid.org/0000-0001-6203-823X) ${ }^{5}$ \\ Sotero Serrate Mengue (https://orcid.org/0000-0002-3349-8541) ${ }^{1}$ \\ Andrêa Damaso Bertoldi (https://orcid.org/0000-0002-4680-3197) ${ }^{6}$ \\ Luiz Roberto Ramos (https://orcid.org/0000-0003-3143-8315) ${ }^{6}$ \\ Mareni Rocha Farias (https://orcid.org/0000-0002-4319-9318) ${ }^{6}$ \\ Tatiane da Silva Dal Pizzol (https://orcid.org/0000-0002-7566-7745) ${ }^{6}$
}

${ }^{1}$ Programa de Pós-

Graduação em

Epidemiologia, Faculdade

de Medicina, Universidade

Federal do Rio Grande do

Sul. R. Ramiro Barcelos

2400, Campus Saúde.

90035-003 Porto Alegre

RS Brasil.karen.costa@

gmail.com

${ }^{2}$ Faculdade de Farmácia, Universidade de Brasília.

Brasília DF Brasil.

${ }^{3}$ Departamento de

Farmácia, Faculdade de

Farmácia, Odontologia e

Enfermagem, Universidade

Federal do Ceará. Fortaleza

CE Brasil.

${ }^{4}$ Núcleo de Assistência

Farmacêutica, Fundação

Oswaldo Cruz (Fiocruz).

Rio de Janeiro RJ Brasil.

${ }^{5}$ Departamento de Política

de Medicamentos e

Assistência Farmacêutica,

Escola Nacional de Saúde

Pública Sérgio Arouca,

Fiocruz. Rio de Janeiro RJ

Brasil.

${ }^{6}$ Grupo PNAUM. Porto

Alegre RS Brasil.
Abstract Objective of this article is to evaluate aspects related to the services provided in SUS pharmacies in Brazil, according to users' perception. Data from the National Survey of Access, Use and Promotion of Rational Use of Medicines carried out between 2013 and 2014 were used. Individuals who obtained drugs from public pharmacies were analyzed. To calculate prevalence estimates, the total number of users of drugs with 95\%CI was used as denominator. From the age group of 20 to 24 years up to 60 to 64 years, there were significant differences between men and women in terms of use of public pharmacies. More than $30 \%$ of people from all socioeconomic classes who did not obtain drugs from SUS pharmacies never thought about this possibility. Not having to wait much time to obtain the medication and a positive evaluation of the opening hours had a strong association with the positive evaluation of users of SUS pharmacies. Opening hours and waiting time are potential barriers in SUS pharmacies. The evaluation of users of SUS was positive, but it pointed to regional differences, and the identification of the magnitude of such differences can contribute to the planning of more effective and equitable policies.

Key words Pharmaceutical Policies, Pharmaceutical Services, Epidemiological Surveys
Resumo Oobjetivo deste artigo é avaliar aspectos relacionados aos serviços prestados nas farmácias do SUS do Brasil, segundo a percepção dos usuários. Utilizou-se dados da Pesquisa Nacional de Acesso, Utilização e Promoção do Uso Racional de Medicamentos, realizada entre 2013 e 2014. Analisou-se indivíduos que obtiveram algum medicamento nas farmácias públicas. Para o cálculo das estimativas de prevalências, foi usado como denominador o total de usuários de medicamentos com IC95\%. A partir da faixa etária de 20 a 24 anos até 60 a 64 anos observa-se diferenças significativas entre homens e mulheres, em relação ao uso de farmácias públicas. Mais de 30\% das pessoas de todas as classes socioeconômica que não obtiveram medicamentos nas farmácias do SUS, nunca pensaram nessa possibilidade. Não costumam esperar para obtenção dos medicamentos e avaliação positiva do horário de funcionamento tiveram uma associação mais forte em relação a avaliação positiva dos usuários das farmácias do SUS. O horário de funcionamento e o tempo de espera são potenciais barreiras nas farmácias do SUS. A avaliação dos usuários que utilizam o SUS é positiva, mas aponta diferenças regionais e a identificação da magnitude dessas pode contribuir na formulação de políticas mais eficazes e equânimes.

Palavras-chave Políticas Farmacêuticas, Serviços Farmacêuticos, Inquéritos Epidemiológicos 


\section{Introdução}

O Sistema Único de Saúde (SUS) é uma política pública avançada e tem como princípios, além do direito à saúde, a universalidade, a equidade e a integralidade. O Estado brasileiro, assumiu desde 1988, na Constituição Federal e posteriormente pela Lei Federal no 8.080 de 1990, a responsabilidade da oferta e financiamento de todos os serviços de saúde. Desde então, diversas políticas têm sido implementadas e operacionalizadas buscando garantir oferta mais eficiente e equitativa ${ }^{1}$.

Os diferentes modelos de sistema de saúde no mundo possuem como um dos seus desafios a promoção de políticas públicas que garantam o acesso de sua população a medicamentos com qualidade e a promoção do seu uso racional ${ }^{2}$. No Brasil, a garantia da Assistência Farmacêutica integral está prevista dentre os direitos da população e dever do Estado.

Estudo nacional, apontou alta aceitabilidade geral dos usuários as farmácias do SUS (> 92\%), no entanto, diferenças regionais indicam a necessidade de direcionar esforços de melhoria da qualidade do atendimento, especialmente nas regiões Norte e Nordeste do país ${ }^{3}$.

A partir da descentralização da Assistência Farmacêutica, os municípios passaram a arcar, em sua grande maioria, com as etapas de seleção, programação, aquisição, armazenamento, distribuição e dispensação de medicamentos ${ }^{4}$. No entanto, os municípios enfrentam distintos problemas, vários deles vinculados ao porte populacional, que limitam e/ou dificultam o exercício da sua função gestora e executora no Sistema, a respeito da garantia do acesso aos medicamentos a população.

Os problemas encontrados envolvem a baixa disponibilidade de medicamentos, que se mostra inversamente associada com a organização da unidade de saúde em geral e da assistência farmacêutica em particular ${ }^{4}$ e a ausência de orientação ao usuário quanto à utilização correta desses produtos ${ }^{5}$.

Este estudo teve como objetivo avaliar, segundo a percepção dos usuários das residentes nas diferentes regiões do país, aspectos relacionados aos serviços prestados nas farmácias públicas no Brasil.

\section{Método}

A Pesquisa Nacional de Acesso, Utilização e Promoção do Uso Racional de medicamentos (PNAUM) tratou-se de um estudo transversal de base populacional baseado em uma amostra probabilística. A coleta dos dados ocorreu entre setembro de 2013 a fevereiro de 2014. As entrevistas foram realizadas face-a-face nas residências, com o registro dos dados em tablets com software desenvolvido especificamente para a aplicação dos questionários.

O instrumento de coleta de dados consistiu em um conjunto de questionários, desenvolvido por um grupo de pesquisadores de Universidades Brasileiras e pode ser consultado no site do componente inquérito PNAUM (http://www.ufrgs.br/ pnaum).

A amostra foi sorteada a partir de 8 domínios definidos, referentes a sexo e idade (incluindo todas as idades da população) que foram replicadas para as 5 grandes regiões do país. O sorteio da amostra foi feito por conglomerados em três estágios: municípios (amostragem sistemática com probabilidade proporcional ao tamanho - unidade amostral primária), setor censitário (dois setores por município selecionados com probabilidade proporcional ao tamanho) e domicílio (sorteados a partir do Cadastro Nacional de Endereços do Censo 2010 do Instituto Brasileiro de Geografia e Estatística (IBGE), considerando apenas aqueles particulares permanentes na zona urbana do território brasileiro) ${ }^{6}$.

Para a estimativa de tamanho da amostra, obtida a partir da Pesquisa Nacional por Amostra de Domicílios - PNAD 2008 (IBGE) ${ }^{7}$, foi considerada uma prevalência de $34,0 \%$ de acesso totalmente gratuito a medicamentos, $44,0 \%$ de acesso pago e o restante de acesso misto. Também foi adotado 0,05 o valor máximo para os coeficientes de variação das estimativas de interesse $e^{6}$.

Ao final foram entrevistadas 41.433 pessoas, em 245 cidades do país, que após ajuste por região, sexo e idade representam os aproximadamente 171 milhões brasileiros residentes nas áreas urbanas do país. Maiores detalhes sobre a amostragem e logística de coleta de dados podem ser encontrados no artigo metodológico da PNAUM ${ }^{6}$.

Para este estudo foram consideradas pessoas que relataram uso de algum medicamento. nos 15 dias anteriores à entrevista, independente do motivo de uso. Destes, $44,9 \%$ obtiveram algum desses medicamentos pelo SUS sendo, $38,4 \%$ das pessoas com 20 anos ou mais.

A pergunta realizada para identificar as pessoas que referiram à obtenção de alguns dos medicamentos em uso no momento da entrevista nas Farmácias do SUS, foi: "Entrevistado obtém algum dos remédios que utiliza no SUS? Sim ou Não". 
Quadro 1. Questões consideradas na análise das Farmácias do Sistema Único de Saúde. PNAUM, Brasil, 2014.

\begin{tabular}{|c|c|}
\hline Questões & Alternativas \\
\hline \multicolumn{2}{|l|}{ Questões respondidas por indivíduos que obtiveram algum medicamento no SUS } \\
\hline $\begin{array}{l}\text { Este local é o mesmo onde o(a) Sr(a) é atendido(a) para tratar seus } \\
\text { problemas de saúde? }\end{array}$ & $\begin{array}{l}\text { ( ) Sim } \\
\text { ( ) Não }\end{array}$ \\
\hline Neste local o(a) $\operatorname{Sr}(a)$ consegue todos os remédios que precisa? & $\begin{array}{l}\text { ( ) Sim } \\
\text { ( ) Não }\end{array}$ \\
\hline Alguma vez faltou algum desses remédios? & $\begin{array}{l}\text { ( ) Sim } \\
\text { ( ) Não }\end{array}$ \\
\hline Com que frequência? & $\begin{array}{l}\text { ( ) Sempre falta } \\
\text { ( ) Às vezes falta } \\
\text { ( ) Quase sempre falta }\end{array}$ \\
\hline $\begin{array}{l}\text { Este local aceita receita de médico particular ou do convênio para retirar os } \\
\text { remédios? }\end{array}$ & $\begin{array}{l}\text { ( ) } \operatorname{Sim} \\
\text { ( ) Não }\end{array}$ \\
\hline Para retirar o(s) remédio(s) precisa participar de algum grupo ou reunião? & $\begin{array}{l}\text { ( ) Sim } \\
\text { ( ) Não }\end{array}$ \\
\hline O remédio pode ser retirado em qualquer dia da semana? & $\begin{array}{l}\text { ( ) Sim } \\
\text { ( ) Não }\end{array}$ \\
\hline Chegar neste local é: & $\begin{array}{l}\text { ( ) Muito difícil } \\
\text { ( ) Um pouco difícil } \\
\text { ( ) Não é difícil }\end{array}$ \\
\hline Este lugar é longe? & $\begin{array}{l}\text { ( ) Sim } \\
\text { ( ) Mais ou menos } \\
\text { ( ) Não }\end{array}$ \\
\hline $\begin{array}{l}\text { Quanto tempo o(a) Sr(a) costuma ficar esperando até ser atendido(a) neste } \\
\text { lugar? }\end{array}$ & $\begin{array}{l}\text { ( ) Não espera } \\
\text { ( ) Um pouco } \\
\text { ( ) Muito tempo }\end{array}$ \\
\hline O horário de funcionamento é: & $\begin{array}{l}\text { ( ) Muito Bom } \\
\text { ( ) Bom } \\
\text { ( ) Regular } \\
\text { ( ) Ruim } \\
\text { ( ) Muito ruim }\end{array}$ \\
\hline O atendimento nesse lugar para retirar os remédios é & $\begin{array}{l}\text { ( ) Muito Bom } \\
\text { ( ) Bom } \\
\text { ( ) Regular } \\
\text { ( ) Ruim } \\
\text { ( )Muito ruim }\end{array}$ \\
\hline \multicolumn{2}{|l|}{ Motivos para não uso das farmácias públicas } \\
\hline Por que não tentou obter no SUS? & $\begin{array}{l}\text { ( ) Porque nunca pensei nisso } \\
\text { ( ) Porque tenho plano de saúde } \\
\text { ( ) Porque é longe } \\
\text { ( ) Porque é demorado } \\
\text { ( ) Porque o atendimento é ruim }\end{array}$ \\
\hline
\end{tabular}


tivamente o serviço de farmácia. Na regressão foi utilizada como variável dependente a pergunta com o desfecho dicotomizado: "O atendimento nesse lugar para retirar os remédios é: Bom/ Muito bom ou Regular/Ruim/Muito Ruim”. A categoria de referência utilizada corresponde às respostas "Muito Bom/Bom" com intervalos de 95\% de confiança (IC95\%). O modelo foi ajustado por região de moradia do entrevistado, sexo e idade.

A pesquisa foi aprovada pelo Comissão $\mathrm{Na}-$ cional de Ética em Pesquisa (CONEP) e pelo Comitê de Ética em Pesquisa da Universidade Federal do Rio Grande do Sul. As entrevistas foram realizadas após leitura do termo de consentimento e assinatura do entrevistado ou seu responsável legal (no caso de incapazes), sendo garantido aos entrevistados sigilo e anonimato.

\section{Resultados}

As mulheres usam mais as farmácias públicas comparado aos homens, exceto nas faixas etárias de 0 a 9 anos. A partir da faixa etária de 20 a 24 anos até 60 a 64 anos são encontradas diferenças estatisticamente significativas no uso desses serviços entre homens e mulheres. A diferença da obtenção de medicamentos entre os sexos na região Norte não é estatisticamente significativa. As regiões onde os usuários mais utilizam os serviços de farmácia do SUS são Sul 26,3\% e Sudeste $25,9 \%$. Não foi observada diferenças no uso das farmácias do SUS segundo os anos de estudo dos indivíduos (Tabela 1).

A Tabela 2 apresenta a avaliação dos usuários dos serviços prestados nas farmácias do públicas. A obtenção de todos os medicamentos que o in-

Tabela 1. Distribuição da amostra e prevalência dos usuários das Farmácias do SUS no Brasil, segundo variáveis sócio demográficas, estratificadas por sexo. PNAUM. Brasil.

\begin{tabular}{|c|c|c|c|c|c|c|}
\hline \multirow{2}{*}{$\begin{array}{c}\text { Características dos } \\
\text { usuários do SUS }\end{array}$} & \multicolumn{2}{|c|}{ Sexo masculino } & \multicolumn{2}{|c|}{ Sexo feminino } & \multicolumn{2}{|c|}{ Total } \\
\hline & $\%^{a}$ & IC95\% & $\%^{a}$ & IC95\% & $\%^{a}$ & IC95\% \\
\hline \multicolumn{7}{|l|}{ Faixa etária } \\
\hline $0-9$ & 17,0 & $14,7-19,7$ & 15,7 & $13,1-18,7$ & 16,4 & $14,3-18,7$ \\
\hline $10-14$ & 12,8 & $9,7-16,7$ & 14,6 & $11,4-18,4$ & 13,7 & $11,2-16,6$ \\
\hline $15-19$ & 5,9 & $4,0-8,6$ & 12,5 & $9,6-16,2$ & 9,3 & $7,3-11,8$ \\
\hline $20-24$ & 6,8 & $4,5-10,1$ & 22,4 & $18,8-26,3$ & 14,8 & $12,4-17,5$ \\
\hline $25-29$ & 11,7 & $7,3-18,2$ & 26,8 & $22,8-31,2$ & 19,2 & $15,9-23,0$ \\
\hline $30-34$ & 10,1 & $7,2-14,1$ & 23,0 & $20,1-26,2$ & 16,8 & $14,5-19,3$ \\
\hline $35-39$ & 15,2 & $11,1-20,5$ & 28,8 & $25,2-32,7$ & 22,7 & $20,0-25,6$ \\
\hline $40-44$ & 17,4 & $14,7-20,4$ & 31,3 & $27,9-35,0$ & 24,7 & $22,2-27,5$ \\
\hline $45-49$ & 18,1 & $15,4-21,2$ & 33,0 & $29,4-36,9$ & 26,0 & $23,4-28,8$ \\
\hline $50-54$ & 22,1 & $19,0-25,5$ & 43,0 & $39,1-47,0$ & 33,8 & $31,0-36,6$ \\
\hline $55-59$ & 28,3 & $24,7-32,1$ & 43,9 & $39,6-48,2$ & 37,0 & $34,2-39,9$ \\
\hline $60-64$ & 37,9 & $34,5-41,4$ & 47,8 & $43,8-51,8$ & 43,5 & $40,5-46,5$ \\
\hline $65-69$ & 43,3 & $38,5-48,3$ & 52,1 & $47,7-56,5$ & 48,3 & $44,5-52,1$ \\
\hline 70 ou mais & 45,6 & $41,7-49,5$ & 48,9 & $45,0-52,8$ & 47,5 & $44,3-50,8$ \\
\hline \multicolumn{7}{|l|}{ Região do país } \\
\hline Norte & 15,5 & $12,1-19,5$ & 21,8 & $17,8-26,4$ & 18,8 & $15,2-22,9$ \\
\hline Nordeste & 14,7 & $12,6-17$ & 25,1 & $22,8-27,4$ & 20,3 & $18,4-22,3$ \\
\hline Sudeste & 19,5 & $16,8-22,4$ & 31,6 & $28,4-35,1$ & 25,9 & $23,2-28,8$ \\
\hline Sul & 20,0 & $17,3-22,9$ & 31,9 & $29,2-34,8$ & 26,3 & $23,7-29,0$ \\
\hline Centro-Oeste & 15,0 & $12,7-17,6$ & 25,1 & $21,8-28,6$ & 20,1 & $17,6-22,8$ \\
\hline \multicolumn{7}{|l|}{ Anos de estudos ${ }^{\mathrm{b}, \mathrm{c}}$} \\
\hline Nunca estudou & 41,6 & $36,0-47,3$ & 44,9 & $41,2-48,7$ & 43,7 & $40,3-47,2$ \\
\hline 1 a 8 anos & 39,0 & $35,1-43,2$ & 45,2 & $42,2-48,3$ & 43,1 & $40,2-46,1$ \\
\hline Mais de 8 anos & 42,2 & $38,2-46,3$ & 44,9 & $42,0-47,7$ & 44,0 & $41,3-46,6$ \\
\hline
\end{tabular}




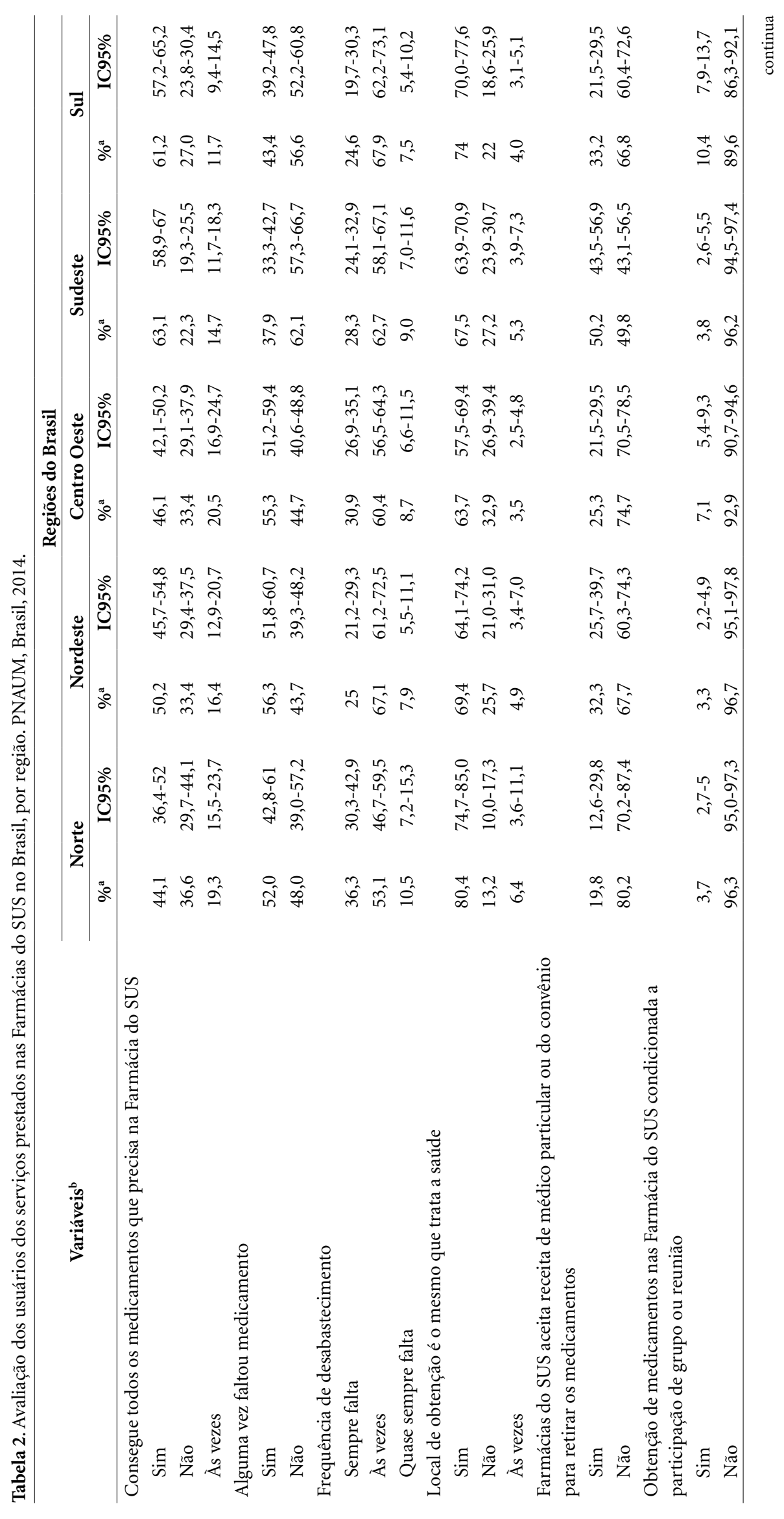




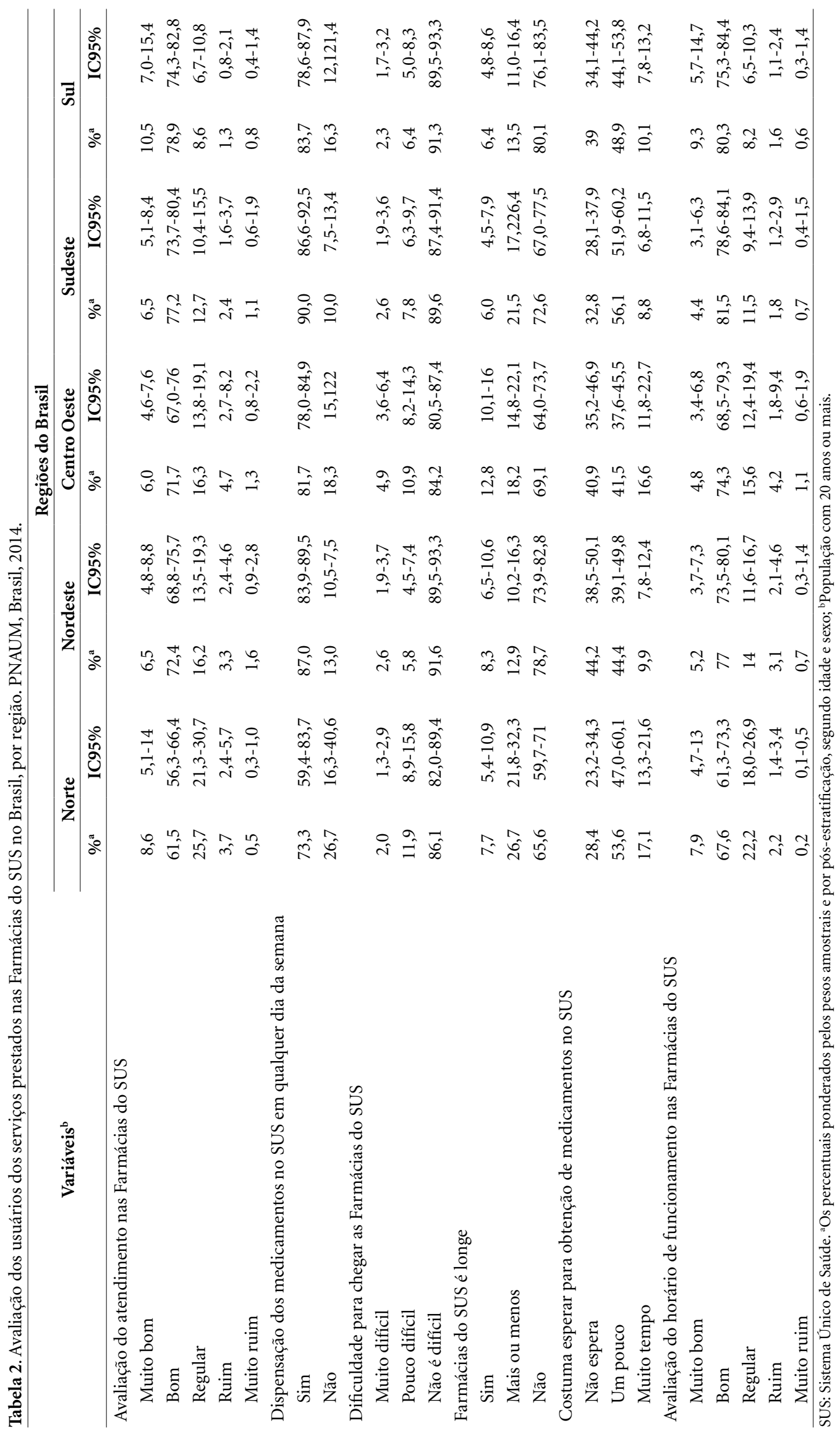


divíduo precisa na farmácia do SUS foi menor na região Norte $(44,1 \%)$ e maior na região Sudeste $(63,1 \%)$. A falta de alguma vez de medicamentos nesses serviços foi menos frequente na região $\mathrm{Su}-$ deste $(37,9 \%)$ e mais frequente na região Nordeste (56,3\%). Verificou-se que sempre falta medicamentos em maior frequência na região Norte (36,3\%).

A região Norte foi a região mais frequente para a obtenção dos medicamentos ser o mesmo local onde o usuário trata da sua saúde $(80,4 \%)$ e o Centro-Oeste a menos frequente $(63,7 \%)$. Na região Sudeste, 50,2\% dos locais de obtenção dos medicamentos aceitam receita do setor privado. A obtenção dos medicamentos condicionada a participação de grupo ou reunião não é observada de forma expressiva na maioria das regiões do país. A maioria dos usuários avaliam o atendimento nas farmácias do SUS como bom e a região Sul $(78,9 \%)$ é a melhor avaliada.

É mais frequente a dispensação de medicamentos em qualquer dia da semana na região Sudeste $(90 \%)$. Chegar ao local de obtenção dos medicamentos no SUS, foi mais difícil $(4,9 \%)$ e mais longe $(12,8 \%)$ para as pessoas da região Centro-Oeste comparada com as demais regiões. $\mathrm{Na}$ região Nordeste as pessoas costumam esperar menos para a obtenção dos medicamentos (44,2\%) e esperam mais tempo os residentes da região Norte $(17,1 \%)$. Costumam avaliar como muito bom o horário de funcionamento das farmácias do SUS 9,3\% das pessoas da região do Sul.

Para avaliar quais fatores influenciam a decisão do usuário em avaliar positivamente o serviço de farmácia, optou-se por usar o modelo de regressão de Poisson. Segundo este modelo a razão de prevalência ajustada por sexo, idade e região dos moradores, as variáveis tempo de espera para obtenção de medicamentos (RP 1,31) e avaliação do horário de funcionamento ( $R P$ $2,65)$, tiveram uma associação forte em relação a avaliação positiva das farmácias do SUS. Conseguir os medicamentos no SUS, poder usar receita de médico particular ou convênios e estar condicionado a participar de grupos para obter os medicamentos mostraram uma associação positiva, porém pequena com a satisfação ainda que estatisticamente significante (RPs de 1,03; 1,03 e 1,03 respetivamente) (Tabela 3 ).

Dentre os motivos daqueles que não obtiveram medicamentos nas farmácias do SUS, nunca pensaram nessa possibilidade mais de $30 \%$ das pessoas de todas as classes socioeconômica, porque não preciso/não quero $(14,6 \%)$ e porque tenho plano de saúde $(7,3 \%)$ maior frequência dentre aqueles das classes A/B. Porque é longe mais referido entre as pessoas da classe C (1,3\%), porque é demorado para mais de $10 \%$ dos indivíduos das classes $\mathrm{C}, \mathrm{D} / \mathrm{E}$, e porque o atendimento é ruim mais frequente entre aqueles das classes D/E (8,4\%) (Figura 1).

\section{Discussão}

O estudo indica diferenças dos usuários das farmácias públicas no Brasil segundo sexo, faixas etárias e regiões do país. De modo geral, as mulheres utilizam mais as farmácias públicas, condizente com outros estudos que verificaram maior proporção de uso dos serviços de saúde entre as mulheres do que os homens ${ }^{8,9}$ e maior utilização de medicamentos ${ }^{10-12}$. Na primeira infância e após a fase adulta a utilização dos medicamentos é superior e tende aumentar gradualmente com a idade em ambos os sexos, também encontrado em outros estudos nacionais e internacionais ${ }^{10,12,13}$ resultando na maior procura pelos medicamentos nas farmácias.

As diferenças observadas na utilização dos serviços das farmácias públicas, podem refletir as desigualdades presentes na utilização de serviços de saúde nas macrorregiões do país. Estudos de Travassos et al. ${ }^{14,15}$ analisaram as disparidades entre as regiões do Brasil, sendo essas marcantes no Nordeste e Sudeste. As pessoas residentes nas regiões Sudeste e Sul apresentaram maior acesso aos serviços quando comparadas aos residentes de outras regiões, conforme descrito por Stopa e colaboradores segundo a Pesquisa Nacional de Saúde $2013^{16}$. Os dados encontrados no presente estudo mostram que os indivíduos das regiões social e economicamente mais desenvolvidas, utilizam com maior frequência as farmácias públicas. Tal dado, sugere que a rede do SUS, estaria mais organizada, de forma a proporcionar melhores serviços nas farmácias públicas nas regiões mais favorecidas, corroborando com outros estudos nacionais ${ }^{17,18}$.

As regiões consideradas mais pobres e/ou menos desenvolvidas possivelmente possuem maior dependência e ineficiência do SUS para acesso aos medicamentos, menos diagnósticos médicos gerados por dificuldades de acesso aos serviços e um provável menor número de locais para obtenção de medicamentos no setor privado do que em outras regiões do país ${ }^{19,20}$.

Não existe diferença no uso das farmácias públicas entre os segmentos menos escolarizados e os mais escolarizados. Em alguma medida, verifica-se que não existe desigualdade, no que se 
Tabela 3. Estimativas da razão de prevalência de avaliação positiva do atendimento do local de obtenção de medicamentos no SUS. PNAUM, Brasil, 2014.

\begin{tabular}{|c|c|c|c|c|c|c|}
\hline \multirow{2}{*}{ Variáveis } & \multicolumn{3}{|c|}{ Análise bruta } & \multicolumn{3}{|c|}{ Análise ajustada } \\
\hline & ${ }^{* *} \mathbf{R P}$ & IC95\% & ${ }^{*} \mathbf{p}$ & $\star * * \mathbf{R P}$ & IC95\% & ${ }^{*} \mathbf{p}$ \\
\hline $\begin{array}{l}\text { Consegue todos os medicamentos que precisa na } \\
\text { Farmácia do SUS }\end{array}$ & 1,13 & $1,08-1,17$ & 0,000 & 1,03 & $1,00-1,07$ & 0,019 \\
\hline $\begin{array}{l}\text { Local de obtenção dos medicamentos no SUS é } \\
\text { longe }\end{array}$ & 0,93 & $0,89-0,98$ & 0,012 & 0,97 & $0,93-1,00$ & 0,116 \\
\hline $\begin{array}{l}\text { Não costuma esperar para obtenção de } \\
\text { medicamentos no SUS }\end{array}$ & 1,79 & $1,61-1,99$ & $<0,001$ & 1,31 & $1,20-1,43$ & $<0,001$ \\
\hline $\begin{array}{l}\text { Avaliação positiva do horário de funcionamento } \\
\text { do local de obtenção de medicamentos no SUS }\end{array}$ & 2,94 & $2,58-3,35$ & $<0,001$ & 2,65 & $2,28-3,06$ & $<0,001$ \\
\hline $\begin{array}{l}\text { Local de obtenção no SUS aceita receita de } \\
\text { médico particular ou do convênio para retirar os } \\
\text { medicamentos }\end{array}$ & 1,07 & $1,03-1,12$ & $<0,001$ & 1,05 & $1,01-1,09$ & 0,005 \\
\hline $\begin{array}{l}\text { Obtenção de medicamentos no SUS } \\
\text { condicionada a participação de grupo ou reunião }\end{array}$ & 1,12 & $1,08-1,16$ & $<0,001$ & 1,03 & $1,00-1,07$ & 0,020 \\
\hline
\end{tabular}

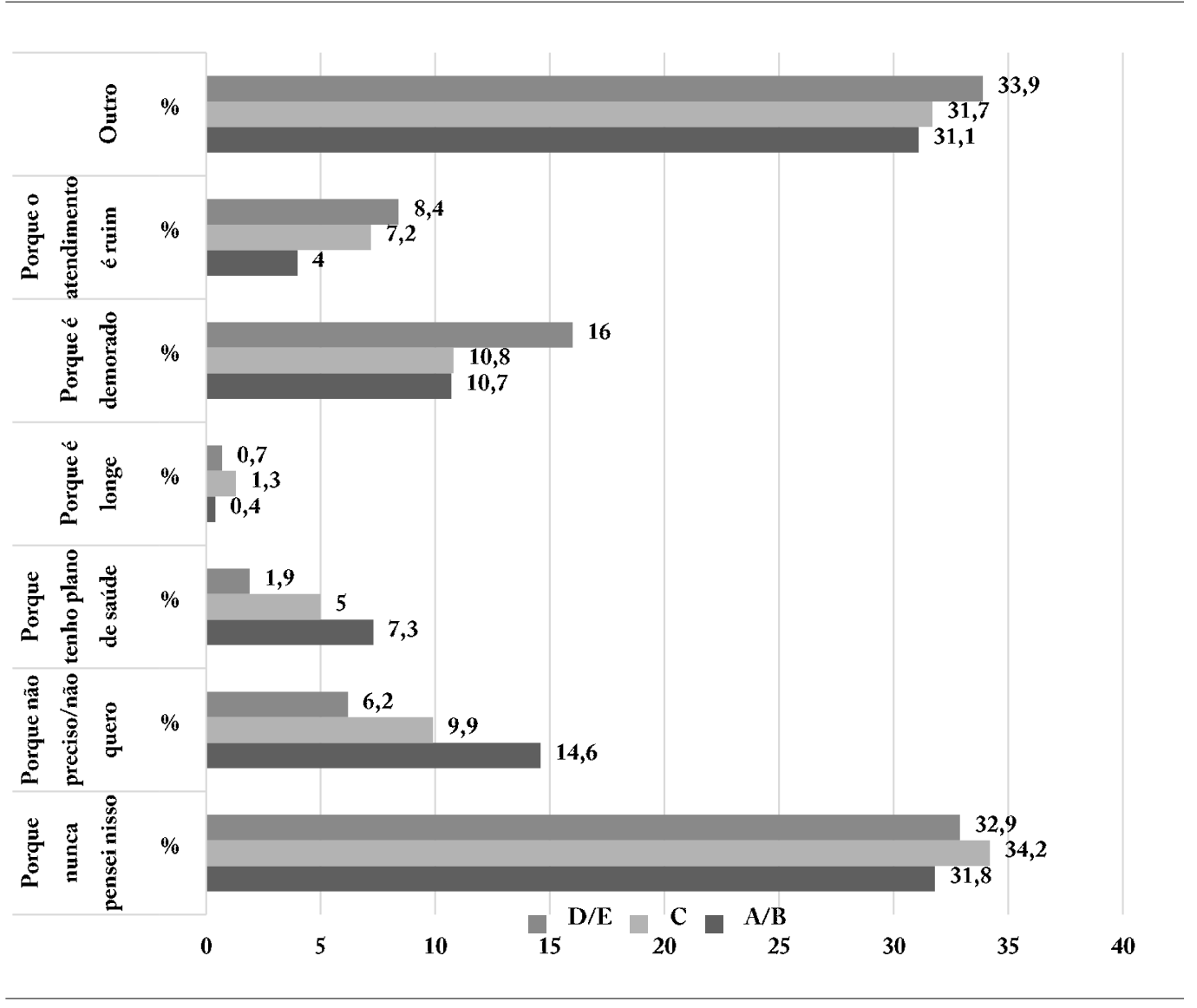

Figura 1. Distribuição dos motivos do não uso das farmácias públicas no Brasil pela população, segundo classificação socioeconômica. PNAUM, Brasil, 2014.

Nota: Os percentuais ponderados pelos pesos amostrais e por pós-estratificação, segundo idade e sexo. População acima de 20 anos. Segundo o Critério de Classificação Econômica Brasil 2013 (CCEB 2013) da Associação Brasileira de Empresas de Pesquisa (ABEP). Disponível em: http://www.abep.org. 
refere ao nível de escolaridade, para a obtenção de algum dos medicamentos que o indivíduo necessita nas farmácias públicas do Brasil. Tal resultado reforça a suposição de que o SUS estaria promovendo a equidade na obtenção dos medicamentos contribuindo com a diminuição das desigualdades entre grupos socioeconômicos.

Já a obtenção de todos os medicamentos que o indivíduo precisa nas farmácias do SUS é desigual entre as regiões do país, sendo maior a obtenção nas regiões mais desenvolvidas. De forma semelhante, a falta de medicamentos e a frequência de desabastecimento é mais frequente nas regiões menos favorecidas, sendo a região Norte com o menor percentual de obtenção de todos os medicamentos que os indivíduos necessitam nas farmácias do SUS. Estudo nacional mostrou que 38\% dos usuários das farmácias da Atenção Primária à Saúde relataram que faltas de medicamentos sempre ocorrem e 35,6\% que, às vezes, os medicamentos não estão disponíveis para a dispensação ${ }^{21}$.

A maior parte do local de obtenção dos medicamentos é o mesmo que o indivíduo trata a sua saúde em todas as regiões do país. Em geral, no âmbito da Atenção Básica, observa-se que as unidades de saúde possuem espaços destinados ao armazenamento e dispensação dos medicamentos. No Brasil 58,8\% das farmácias estavam implantadas em centros/unidades de saúde, 14,6\% em Postos de Saúde e 13,4\% em farmácias de edificação independente ${ }^{21}$. Independente se as farmácias estão localizadas em unidades de saúde ou em edificação exclusivas, devem dispor de infraestrutura física, recursos humanos e materiais que permitam a melhor integração entre os serviços de saúde e o desenvolvimento das atividades da assistência farmacêutica ${ }^{22,23}$.

A receita médica pode ser considerada uma barreira para a obtenção dos medicamentos. As farmácias públicas da região Norte aceitam menos as receitas de médicos particular ou de convênio, oposto verificado na região Sudeste.

$\mathrm{Na}$ região Sul é mais frequente condicionar a obtenção de medicamentos a participação de grupos ou reunião nas unidades de saúde. Paniz et al. ${ }^{24}$ verificaram em inquérito domiciliar realizado na região Sul, que a participação em grupos nas UBS, esteve associada a um maior acesso entre os adultos dessa região, perdendo a significância estatística entre os adultos da Região Nordeste o que pode ser influenciado pelo modelo de atenção adotado e pelos diferentes procedimentos utilizados pelas unidades quanto à participação em grupos, prescrição e fornecimento de medicamentos de uso contínuo ${ }^{24}$.
Dentre as pessoas que obtém medicamentos nas farmácias do SUS, a maioria avalia como bom e muito bom o atendimento nesses locais em todas as regiões, o que corrobora com estudo de Soeiro et al. ${ }^{25}$ onde se identificou que a satisfação dos usuários com o atendimento nas farmácias da atenção básica se apresentou como fator relevante na satisfação geral do usuário ${ }^{25}$. Os usuários satisfeitos tendem a aderir ao tratamento prescrito, a fornecer informações importantes para o provedor e a continuar utilizando os serviços de saúde e tendem a ser mais propensos a uma melhor qualidade de vida ${ }^{25,26}$.

Em todas as regiões do país é mais frequente as pessoas referirem como não é difícil chegar e não é longe as farmácias do SUS. Estudo nacional que avaliou o acesso aos medicamentos na atenção primária no Brasil mostrou que a maioria dos usuários declarou ser fácil ou muito fácil chegar à UBS e, por outro lado, quase um quarto dos usuários relatou que a UBS era longe de sua residência ${ }^{27}$. Outro estudo nacional encontrou desigualdades regionais na acessibilidade geográfica das farmácias públicas, apontando que a distribuição geográfica das farmácias públicas pode melhorar, especialmente nas regiões Norte e Centro-Oeste do país ${ }^{3}$.

A avaliação positiva da população sobre as farmácias públicas no Brasil está relacionada com a maior proporção das pessoas referirem não esperar ou esperar um pouco para a obtenção dos medicamentos nas farmácias do SUS em todas as regiões do país. Segundo publicação do Ministério da Saúde entre os usuários dos serviços farmacêuticos da atenção primária o tempo de espera nas farmácias até o atendimento é um problema para $33,1 \%$ dos usuários ${ }^{21}$.

De forma semelhante, no presente estudo dentre aqueles que conseguiram obter medicamentos no SUS, o horário de funcionamento das farmácias públicas foi avaliado positivamente pelos usuários. Na Atenção Primária, 84,7\% dos usuários avaliaram ser muito bom/bom o horário de funcionamento das UBS, com maior proporção na Região Sudeste $(88,4 \%)$ e a menor na Região Norte $(79,4 \%)$, apresentando diferenças estatísticas significantes entre regiões e, ainda, para $28,3 \%$ dos usuários o horário de funcionamento é um dos itens que poderia ser melhorado nas farmácias públicas ${ }^{21}$.

Em síntese, as farmácias públicas são mais bem avaliadas pelos usuários quando esses não esperam para obter os medicamentos que necessitam e quando avaliam positivamente o horário de funcionamento das farmácias. 
Apesar da relevância das políticas farmacêuticas no país, dentre os indivíduos que não obtiveram medicamentos nas farmácias públicas, para a maioria, nunca foi uma opção obter medicamentos pelo SUS. Além desse principal motivo, porque não precisa ou não quero também é relatado com maior frequência dentre aqueles com maior poder aquisitivo e os que possuíam plano de saúde, como esperado. Esse fato, pode sugerir que parcela da população brasileira não conhece as políticas de acesso a medicamentos no país e/ ou não tem qualquer interesse em obter os medicamentos pela provisão nas farmácias públicas.

Também se verificou que não é considerada uma barreira importante para os brasileiros que não obtêm medicamentos nas farmácias públi- cas, a distância e a qualidade do atendimento, independente das classes econômicas.

Uma das limitações desse estudo é o fato de não ter sido perguntado o número de vezes que os usuários buscaram as farmácias públicas e o período de uso, o que pode ter influenciado nos resultados. Os dados apresentados podem estar subestimados por restrições de memória, uma vez que são autorreferidas pelos usuários. Ainda, devido o tipo de estudo, não se pode estabelecer a temporalidade dos fatores associados.

Em resumo, os resultados deste trabalho corroboram com a definição de Hart sobre a lei de cuidados inversos "[...] em que a disponibilidade por uma boa assistência médica tende a variar inversamente com a necessidade da população atendida"28.

\section{Colaboradores}

KS Costa, AV Zaccolo, NUL Tavares e SS Mengue participaram da concepção da comunicação, análise e interpretação dos dados, redação e revisão do conteúdo. MA Oliveira, PSD Arrais e VL Luiza participaram da revisão do conteúdo. Todos os autores aprovaram a versão a ser publicada.

\section{Agradecimentos}

Ao Departamento de Assistência Farmacêutica e Insumos Estratégicos e ao Departamento de $\mathrm{Ci}$ ência e Tecnologia da Secretaria de Ciência, Tecnologia e Insumos Estratégicos do Ministério da Saúde pelo apoio financeiro.

Este trabalho foi realizado com o apoio da Coordenação de Aperfeiçoamento de Pessoal de Nível Superior - Brasil (CAPES), por meio da bolsa de pós-doutorado da Dra. Karen Sarmento Costa. Rogério Borges e Andréia Turmina Fontanella agradecem à equipe de apoio estatístico do Programa de Pós-Graduação em Epidemiologia da Universidade Federal do Rio Grande do Sul. 


\section{Referências}

1. Medici AC. Propostas para melhorar a cobertura, a eficiência e a qualidade no setor saúde. In: Bacha EL, Schwartzman S, organizadores. Brasil: A Nova Agenda Social. Rio de Janeiro: Livros Técnicos e Científicos; 2011. p. 364.

2. World Health Organization (WHO). How to Develop and Implement a National Drug Policy - WHO Policy Perspectives on Medicines No 6. Geneva: WHO; 2003.

3. Oliveira MA, Luiza VL, Tavares NUL, Mengue SS, Arrais PSD, Farias MR, Dal Pizzol TS, Ramos LR, Bertoldi AD. Access to medicines for chronic diseases in Brazil: a multidimensional approach. Rev Saúde Pública 2016; 50(Supl. 2):6s.

4. Mendes LV, Campos MR, Chaves GC, Silva RM da, Freitas PS, Costa KS, Luiza VL. Disponibilidade de medicamentos nas unidades básicas de saúde e fatores relacionados: uma abordagem transversal. Saúde Debate 2014; 38(Supl):109-123.

5. Oliveira LCF, Assis MMA, Barboni AR. Pharmaceutical Assistance in the Basic Units of Health: from the National Drug Policy to the Basic Attention to Health. Cien Amp Saúde Colet 2010; 15:3561-3567.

6. Mengue SS, Bertoldi AD, Boing AC, Tavares NUL, Pizzol TSD, Oliveira MA, Arrais PSD, Ramos LR, Farias MR, Luiza VL, Bernal RTI, Barros AJD. National Survey on Access, Use and Promotion of Rational Use of Medicines (PNAUM): household survey component methods. Rev Saúde Pública 2016; 50(Supl. 2):4s.

7. Instituto Brasileiro de Geografia e Estatística (IBGE). Pesquisa nacional por amostra de domicílios. Rio de Janeiro: IBGE; 2008.

8. Gontijo MF, Ribeiro AQ, Klein $\mathrm{CH}$, Rozenfeld $\mathrm{S}$ Acurcio FA. Uso de anti-hipertensivos e antidiabéticos por idosos: inquérito em Belo Horizonte, Minas Gerais, Brasil. Cad Saúde Pública 2012; 28(7):1337-1346.

9. Obermeyer CM, Price K, Schulein M, Sievert LL, Anderton DL. Medication use and gender in Massachusetts: results of a household survey. Health Care Women Int 2007; 28(7):593-613.

10. Bertoldi AD, Barros AJ, Hallal PC, Lima RC. Utilização de medicamentos em adultos: prevalência e determinantes individuais. Rev Saúde Pública 2004; 38(2):228-238

11. Carvalho MF, Pascom ARP, Souza-Junior PRB, Damacena GN, Szwarcwald CL. Utilization of medicines by the Brazilian population, 2003. Cad Saúde Pública 2005; 21 (Supl. 1):S100-S108.

12. Costa KS, Barros MBA, Francisco PMSB, Cesar CLG, Goldbaum M, Carandina L. Utilização de medicamentos e fatores associados: um estudo de base populacional no Município de Campinas, São Paulo, Brasil. Cad Saúde Pública 2011; 27(4):649-658.

13. Morgan TK, Williamson M, Pirotta M, Stewart K, Myers SP, Barnes J. A national census of medicines use: a 24-hour snapshot of Australians aged 50 years and older. Med J Aust 2012; 196(1):50-53.

14. Travassos C, Viacava F, Fernandes C, Almeida CM. Desigualdades geográficas e sociais na utilização de serviços de saúde no Brasil. Cien Saude Colet 2000; 5(1):133-149.
15. Travassos C, Oliveira EXG, Viacava F. Desigualdades geográficas e sociais no acesso aos serviços de saúde no Brasil: 1998 e 2003. Cien Saude Colet 2006; 11(4):975-986.

16. Stopa SR, Malta DC, Monteiro CN, Szwarcwald CL, Goldbaum M, Galvão Cesar CL. Acesso e uso de serviços de saúde pela população brasileira, Pesquisa Nacional de Saúde 2013. Rev Saúde Pública 2017; 51(Supl.1):3s.

17. Paniz VMV, Fassa AG, Facchini LA, Bertoldi AD, Piccini RX, Tomasi E, Thumé E, Silveira DS, Siqueira FV, Rodrigues MA. Acesso a medicamentos de uso contínuo em adultos e idosos nas regiões Sul e Nordeste do Brasil. Cad Saúde Pública 2008; 24(2):267-280.

18. Tavares NUL, Luiza VL, Oliveira MA, Costa KS, Mengue SS, Arrais PSD, Ramos LR, Farias MR, Dal Pizzol TS, Bertoldi AD. Free access to medicines for the treatment of chronic diseases in Brazil. Rev Saúde Pública 2016; 50(Supl. 2):7s.

19. Bertoldi AD, Pizzol TSD, Ramos LR, Mengue SS, Luiza VL, Tavares NUL, Farias MR, Oliveira MA, Arrais PSD. Sociodemographic profile of medicines users in Brazil: results from the 2014 PNAUM survey. Rev Saúde Pública 2016; 50(Supl. 2):5s.

20. Andrade MV, Noronha KVM de S, Menezes R de M Souza MN, Reis CB, Martins DR, Gomes L. Desigualdade socioeconômica no acesso aos serviços de saúde no Brasil: um estudo comparativo entre as regiões brasileiras em 1998 e 2008. Econ Apl 2013; 17(4):623645.

21. Brasil. Ministério da Saúde (MS). Secretaria de Ciência, Tecnologia e Insumos Estratégicos. Componente Avaliação dos Serviços de Assistência Farmacêutica Básica: resultados. Brasília: MS; 2017.

22. Brasil. Ministério da Saúde (MS). Secretaria de Ciência, Tecnologia e Insumos Estratégicos, Departamento de Assistência Farmacêutica. Diretrizes para estruturação de farmácias no âmbito do Sistema Único de Saúde. Brasília: MS; 2009.

23. Leite SN, Manzini F, Álvares J, Guerra Junior AA, Costa EA, Acurcio FA, Guibu IA, Costa KS, Karnikowski MGO, Soeiro OM, Farias MR. Infrastructure of pharmacies of the primary health care in the Brazilian Unified Health System: Analysis of PNAUM - Services data. Rev Saúde Pública 2017; 51(Supl. 2 ):13s.

24. Paniz VMV, Fassa AG, Facchini LA, Bertoldi AD, Piccini RX, Tomasi E, Thumé E, Silveira DS, Siqueira FV, Rodrigues MA. Access to continuous-use medication among adults and the elderly in South and Northeast Brazil. Cad Saúde Pública 2008; 24(2):267-280.

25. Soeiro OM, Tavares NUL, Nascimento Júnior JM, Guerra Junior AA, Costa EA, Acurcio FA, Guibo IA, Álvares J, Karnikowski MGO, Leite SN, Costa KS. Patient satisfaction with pharmaceutical services in Brazilian primary health care. Rev Saúde Pública 2017; 51(Supl. 2):21s.

26. Baron-Epel O, Dushenat M, Friedman N. Evaluation of the consumer model: relationship between patients' expectations, perceptions and satisfaction with care. Int J Qual Health Care 2001; 13(4):317-323. 
27. Álvares J, Guerra Junior AA, Araújo V, Almeida AM, Dias CZ, Ascef BO, Costa EA, Guibu IA, Soeiro OM, Leite SN, Karnikowski MGO, Costa KS, Acurcio FA. Access to medicines by patients of the primary health care in the Brazilian Unified Health System. Rev Saúde Pública 2017; 51(Supl. 2):20s.

28. Hart TJ. The inverse care law. Lancet 1971; 297(7696):405-412.

Artigo apresentado em 08/07/2017

Aprovado em 27/11/2018

Versão final apresentada em 29/11/2018 


\section{ERRATA}

p. 3163,

onde se lê:

Karen Sarmento Costa (http://orcid.org/0000-0002-2218-6024) ${ }^{1}$

Anamaria Vargas Zaccolo (https://orcid.org/0000-0001-6195-9301) ${ }^{1}$

Noemia Urruth Leão Tavares (https://orcid.org/0000-0001-6180-7527) ${ }^{2}$

Paulo Sérgio Dourado Arrais (https://orcid.org/0000-0002-4502-8467) ${ }^{3}$

Vera Lucia Luiza (https://orcid.org/0000-0001-6245-7522) ${ }^{4}$

Maria Auxiliadora Oliveira (https://orcid.org/0000-0001-6203-823X) ${ }^{5}$

Sotero Serrate Mengue (https://orcid.org/0000-0002-3349-8541) ${ }^{1}$

\section{leia-se:}

Karen Sarmento Costa (http://orcid.org/0000-0002-2218-6024) ${ }^{1}$

Anamaria Vargas Zaccolo (https://orcid.org/0000-0001-6195-9301) ${ }^{1}$

Noemia Urruth Leão Tavares (https://orcid.org/0000-0001-6180-7527) ${ }^{2}$

Paulo Sérgio Dourado Arrais (https://orcid.org/0000-0002-4502-8467) ${ }^{3}$

Vera Lucia Luiza (https://orcid.org/0000-0001-6245-7522) ${ }^{4}$

Maria Auxiliadora Oliveira (https://orcid.org/0000-0001-6203-823X) ${ }^{5}$

Sotero Serrate Mengue (https://orcid.org/0000-0002-3349-8541) ${ }^{1}$

Andrêa Damaso Bertoldi (https://orcid.org/0000-0002-4680-3197) ${ }^{6}$

Luiz Roberto Ramos (https://orcid.org/0000-0003-3143-8315) ${ }^{6}$

Mareni Rocha Farias (https://orcid.org/0000-0002-4319-9318) ${ }^{6}$

Tatiane da Silva Dal Pizzol (https://orcid.org/0000-0002-7566-7745) ${ }^{6}$

\section{onde se lê:}

${ }^{1}$ Programa de Pós-Graduação em Epidemiologia, Faculdade de Medicina, Universidade Federal do Rio Grande do Sul. R. Ramiro Barcelos 2400, Campus Saúde. 90035-003 Porto Alegre RS Brasil. karen.costa@gmail.com

${ }^{2}$ Faculdade de Farmácia, Universidade de Brasília. Brasília DF Brasil.

${ }^{3}$ Departamento de Farmácia, Faculdade de Farmácia, Odontologia e Enfermagem, Universidade Federal do Ceará. Fortaleza CE Brasil.

${ }^{4}$ Núcleo de Assistência Farmacêutica, Fundação Oswaldo Cruz.

${ }^{5}$ Departamento de Política de Medicamentos e Assistência Farmacêutica, Escola Nacional de Saúde Pública Sérgio Arouca, Fundação Oswaldo Cruz. Rio de Janeiro RJ Brasil.

\section{leia-se:}

${ }^{1}$ Programa de Pós-Graduação em Epidemiologia, Faculdade de Medicina, Universidade Federal do Rio Grande do Sul. R. Ramiro Barcelos 2400, Campus Saúde. 90035-003 Porto Alegre RS Brasil. karen.costa@gmail.com

${ }^{2}$ Faculdade de Farmácia, Universidade de Brasília. Brasília DF Brasil.

${ }^{3}$ Departamento de Farmácia, Faculdade de Farmácia, Odontologia e Enfermagem, Universidade Federal do Ceará. Fortaleza CE Brasil.

${ }^{4}$ Núcleo de Assistência Farmacêutica, Fundação Oswaldo Cruz (Fiocruz). Rio de Janeiro RJ Brasil.

${ }^{5}$ Departamento de Política de Medicamentos e Assistência Farmacêutica, Escola Nacional de Saúde Pública Sérgio Arouca, Fiocruz. Rio de Janeiro RJ Brasil.

${ }^{6}$ Grupo PNAUM. Porto Alegre RS Brasil. 